
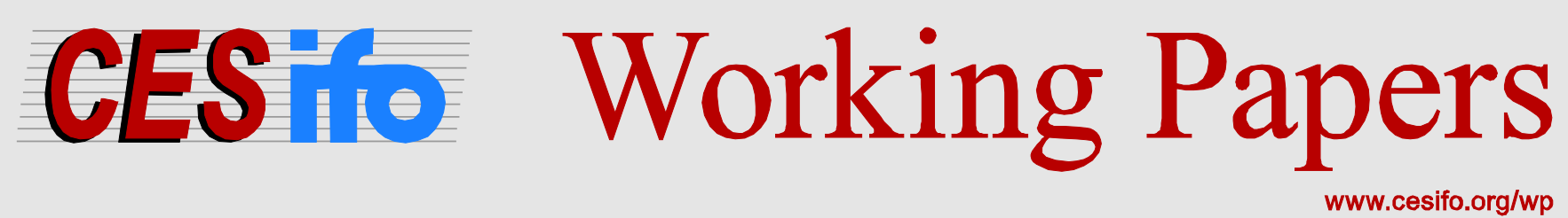

\title{
Risk-Taking-Neutral Background Risk
}

\author{
Guenter Franke \\ Harris Schlesinger \\ Richard C. Stapleton
}

\author{
CESIFO WORKING PAPER NO. 4070 \\ CATEGORY 12: EMPIRICAL AND THEORETICAL METHODS \\ JANUARY 2013
}

An electronic version of the paper may be downloaded

- from the SSRN website: Www.SSRN.com

- from the RePEc website: $\quad$ www.RePEc.org

- from the CESifo website: www.CESifo-group.org/wp

\section{CESifo}




\title{
Risk-Taking-Neutral Background Risk
}

\begin{abstract}
We define a class of risk-taking-neutral (RTN) background risks. These background risks have the property that they will not alter decisions made with respect to another risk, for individuals with HARA utility. If we wish to compare a decision made with and without some exogenous background risk, it is often easier to compare the decision made to one made with a RTN background risk. We use this methodology to prove and extend a well-known theorem about dynamic investment strategy, due to Mossin (1968a). We also use this methodology to analyze investment behavior in the presence of an income tax as well as to analyze investment behavior in the presence of particular types of background risks.
\end{abstract}

JEL-Code: D810, G110.

Keywords: background risk, HARA utility, income tax, portfolio choice, risk vulnerability.

\author{
Guenter Franke \\ University of Konstanz \\ Konstanz / Germany \\ guenter.franke@uni-konstanz.de
}

\author{
Harris Schlesinger \\ University of Alabama \\ Tuscaloosa / Alabama / USA \\ hschlesi@cba.ua.edu
}

\author{
Richard C. Stapleton \\ University of Manchester \\ Manchester / United Kingdom \\ richard.stapleton1@btinternet.com
}

November 21, 2012

We thank seminar participants at Imperial College, at the Universities of Bonn, Canterbury, Chile, Konstanz and Pennsylvania, as well as Francesca Barigozzi, Mario Brandtner and Jens Jackwerth, for helpful comments on an earlier draft of this paper. 


\section{Introduction}

Consider an individual who must make an economic decision in the face of risk. For example, the individual might be an investor deciding on how to allocate wealth between a risky and a risk-free asset. Alternatively, the individual might be deciding on how to insure or how to otherwise hedge a risky asset. Typically, such a decision is modeled in isolation, where there is only the one source of risk. For the sake of clarity, we will refer to this risk as the "primary risk." However, more realistically, there are other risks that are also faced by the individual. One type of such risk is often referred to as a "background risk," meaning that there is no market for trading directly on this second risk. One question that has been given considerable attention in the literature is "how does the presence of this background risk affect behavior towards the primary risk?" Obviously, many types of dependence between the risks might be indirectly treated via trading on the primary risk. For example, contracts on the primary risk might partially mitigate effects of the background risk via "cross hedging" techniques. However, even in cases where such techniques are not possible, such as when the two risks are independent, it is now well known that a background risk can still affect decisions about the treatment of the primary risk. ${ }^{1}$

\footnotetext{
${ }^{1}$ This line of research began with Kihlstrom et al. (1981), Ross (1981) and Nachman (1982). These papers all considered one individual who was more risk averse than another with resepect to the primary risk and examined whether the individual remained more risk averse (with respect to the primary risk) in the presence of such a background risk. Doherty and Schlesinger (1983) showed how such a background risk might affect an individual's decision towards the primary risk. Good summaries of how such background
} 
For example, one might hypothesize that an independent additive zero-mean background risk will lead to less risk taking with respect to the primary risk. However, as shown by Gollier and Pratt (1996), risk aversion alone is not sufficient to guarantee such behavior. Preferences that do guarantee such behavior are labeled as "risk vulnerable" by Gollier and Pratt (1996), who also derive the tedious necessary and sufficient conditions that lead to such behavior. Luckily, relevant sufficient conditions are much easier to satisfy. A few papers have looked at cases where the two risks are not independent, but these cases are usually quite restrictive. ${ }^{2}$ As another illustration, consider investment decisions modelled in a world without taxes. How would the inclusion of an income tax affect investment strategy?

In this paper, we define a class of "risk-taking-neutral" (RTN) background risks for an individual with preferences that satisfy "hyperbolic absolute risk aversion" (HARA). For an individual with HARA utility, a RTN background risk will not affect decisions made about the primary risk. Confining our analysis to the HARA class is not too severe of a restriction, since the closure of the HARA class of utility includes most all of the commonly used utility functions, including constant absolute risk aversion (CARA), constant relative risk aversion (CRRA) and quadratic utility. The importance of the HARA class within finance includes it's equivalence to the set of utility functions allowing for two-fund separation in portfolio choice, as shown by Cass and Stiglitz (1970). Moreover, the HARA class of utility allows for dynamic portfolio choices to be made somewhat myopically, "as if" future period returns were all risk-free. See Mossin (1968a) and Gollier (2001). Indeed, we will prove Mossin's (1968a) main result later in the paper, as an example of a risk-taking-neutral background risk.

Although our class of RTN background risks is fairly simple to construct, such background risks might not resemble many exogenous background risk that occurs naturally within an economy. However, these RTN background risks can often serve as a benchmark. In particular, in many situations, a canonical type of background risk can be compared to a member of the RTN class in such a way as to predict qualitative changes in risk-taking behavior

risks embed into economic and financial decisions can be found in Campbell and Viceira (2002) and Gollier (2001).

${ }^{2}$ See, for example, Dana and Scarsini (2007). 
towards the primary risk. Indeed, it might be more difficult to compare this canonical background risk directly to a world no background risk. The comparison with a suitably chosen RTN background risk might make the economic analysis much simpler.

We begin the next section with a description of our class of RTN background risks within the class of HARA utility. We next show how several examples of background risks can be analyzed via comparison to our RTN class of background risks. In particular, we examine portfolio choice in a few different settings.

Section 3 first shows how a famous result about dynamic portfolio choice, due to Mossin (1968a), follows in straightforward manner using RTN background risk. We also show how Mossin's result can be extended to certain cases in which the future risk-free rate is unknown at the time current portfolio decisions must be made. Section 4 examines the effect of an income tax on optimal portfolio choice and shows how it can be easily modelled using our RTN background risk. The result is also extended to a world in which the tax rate is random in the sense of being unknown at the time investment decisions must be made.

In section 5, we consider an independent, additive, zero-mean background risk, as in Gollier and Pratt (1996). By choosing an appropriate RTN background risk, which by construction does not affect investment decisions, we can easily show the logic behind the Gollier and Pratt result. In section 6, we provide a more general result showing how the RTN class of background risks can be used as a benchmark to predict qualitative changes in investment when wealth is subjected to an exogenous background risk that is linearly dependent on one's portfolio wealth. We conclude by summarizing our results as well as noting some of their limitations.

\section{Risk-Taking-Neutral Background Risk}

Consider a risk-averse individual with random wealth $\widetilde{x}$ (the "primary risk") who maximizes her expected utility of terminal wealth. We assume that utility belongs to the so-called hyperbolic-absolute-risk-aversion (HARA) class 
of utility, where we can express utility as

$$
u(x)=\frac{1}{B-1}(A+B x)^{1-\frac{1}{B}},
$$

where $B \neq 0, B \neq 1$ and $A+B x>0$. Risk tolerance for this class of utility is linear:

$$
\frac{-u^{\prime}(x)}{u^{\prime \prime}(x)}=A+B x
$$

Note that this class of utility includes constant relative risk aversion $(A=0)$ and quadratic utility $(B=-1)$. If $A$ is positive [negative], preferences exhibit increasing [decreasing] relative risk aversion. If $B$ is positive [negative], preferences exhibit decreasing [increasing] absolute risk aversion. ${ }^{3}$

We assume that the random wealth $\widetilde{x}$ is endogenous in that the individual can engage in market activity affecting the distribution of terminal wealth. For the sake of concreteness, we will consider the so-called standard portfolio problem: the individual allocates her wealth between a risky asset and a risk-free asset. We assume that the expected risky return is higher than the risk-free return. From standard portfolio-choice analysis, we know that a risk-averse individual always invests a positive amount in the risky asset when its expected payoff per dollar invested is higher than the payoff on the risk-free asset. Moreover, a more risk-averse individual would always invest less in the risky asset, ceteris paribus.

We now suppose that the investor faces a second risk $\widetilde{y}$, the so-called "background risk" for which there is no market available for trading and/or hedging. Final wealth is denoted as $\widetilde{x}+\widetilde{y}$. The question addressed in this paper is whether or not we can predict that the individual take less [or more] risk with respect to the primary risk $\widetilde{x}$ in the market in the presence of background risk $\widetilde{y}$.

To facilitate answering such a question, we define the class of risk-takingneutral (RTN) background risks to be any background risk of the following form

$$
\widetilde{z}(x)=(k+\widetilde{\varepsilon})\left(\frac{A}{B}+x\right),
$$

\footnotetext{
${ }^{3}$ It also is straightforward to show, using L'Hospital's rule, that such utility approaches constant-absolute-risk-aversion (CARA) utility as $B \rightarrow 0, u(x)=-e^{-\theta x}$, with $\theta=\frac{1}{A}$. Also, utility approaches $\log$ utility, $u(x)=\ln x$, if $A=0$ and $B \rightarrow 1$.
} 
where $k \in \mathbb{R}$ and the random variable $\widetilde{\varepsilon}$ is statistically independent of the random variable $\widetilde{x}$, with $E \widetilde{\varepsilon}=0$, where $E$ denotes the expectation operator. Also, since HARA requires $A+B(x+z)>0$, we assume that $1+k+\varepsilon>0$ for all values of $\varepsilon$. Note that we allow for the possibility that $\widetilde{\varepsilon}$ is degenerate, with variance zero. Although $\widetilde{\varepsilon}$ and $\widetilde{x}$ are statistically independent by assumption, $\widetilde{\varepsilon}$ and $\widetilde{z}$ are statistically dependent by construction. Indeed, there is a particular type of linear dependence between $\widetilde{z}(x)$ and $\widetilde{x}$.

It is important to note that our construction of $\widetilde{z}$ is parametric, based on the parameters in the decision maker's utility function $u$. It is also important to note that only the ratio $\frac{A}{B}$ matters in our construction. This is important since our definition of HARA in (1) is unique only up to an affine transformation of $u$.

To see the effect of $\widetilde{z}$ on decisions made about the primary risk $\widetilde{x}$, we consider the so-called derived utility function ${ }^{4}$ for an arbitrary background risk $\widetilde{z}$ :

$$
v(x) \equiv E u(x+\widetilde{z}) .
$$

For $\widetilde{z}$ belonging to the RTN class of background risk, we obtain

$$
\left.v(x)=E\left[\frac{1}{B-1}((A+B x)(1+k+\widetilde{\varepsilon}))^{1-\frac{1}{B}}\right]=E(1+k+\widetilde{\varepsilon})\right)^{1-\frac{1}{B}} \cdot u(x) .
$$

In other words, the derived utility $v(x)$ is a positive constant, $E(1+k+\widetilde{\varepsilon})^{1-\frac{1}{B}}$, multiplied by utility $u(x)$. Since $\widetilde{\varepsilon}$ is statistically independent of $\widetilde{x}$, decisions made about the primary risk $\widetilde{x}$ in the presence of background risk are identical to decisions on $\widetilde{x}$ without background risk, but using the derived utility function $v$ in place of $u$. That is

$$
E v(\widetilde{x})=E u(\widetilde{x}+\widetilde{z})=E(1+k+\widetilde{\varepsilon})^{1-\frac{1}{B}} \cdot E u(\widetilde{x}) .
$$

In any generic type of decision problem about $\widetilde{x}$, we can interpret the firstorder conditions as setting marginal benefits equal to marginal costs, for changes in the decision variable, where benefits and costs are given in terms

\footnotetext{
${ }^{4}$ See Kihlstrom et al. (1981), who refer to this function as the indirect utility function, and Nachman (1982). Note also that this background risk contains a mixture of an additive and multiplicative background risk. See, for example, Franke et al. (2011).
} 
of marginal utility. Since both marginal benefits and marginal costs are simply scaled by the common multiplicative factor $E(1+k+\widetilde{\varepsilon})^{1-\frac{1}{B}}$ in the presence of $\widetilde{z}$, the optimal decision remains unchanged. It is important to note that we are not claiming that preferences are unaffected by $\widetilde{z}$. If $E(1+k+\widetilde{\varepsilon})^{1-\frac{1}{B}}<1$, then the individual is worse off when background risk $\widetilde{z}$ is present. If the inequality is reversed, the individual is better off. However, decisions made about $\widetilde{x}$ are not affected whether $\widetilde{z}$ is present or not, hence our terminology "risk-taking-neutral background risk."

Note that the RTN class defined in (3) is not an exclusive list of all the background risks with no effect on decision making. As one example consider our RTN background risk for quadratic utility $(B=-1)$, which gives a multiplicative affine transformation. But with quadratic utility, an independent zero-mean background risk yields an additive dead weight loss to expected utility. Thus it also does not affect economic decisions made on $\widetilde{x}$. The point is that our definition of the RTN class of background risks is not an exclusive set of background risks that yield no effect on decisions. However, as we demonstrate below, the RTN class defined here can prove quite useful in analyzing decisions made under uncertainty.

The types of background risk $\widetilde{y}$ that a decision might face will not typically belong to the class of risks given by (3) above, although it certainly might. In the case where $\widetilde{y}$ belongs to the RTN class our conclusion is simple: the individuals makes the same choice with or without the background risk $\widetilde{y}$. But in the more typical case where the background risk $\widetilde{y}$ does not belong to the RTN class, we will show how an appropriately chosen RTN background risk can provide a benchmark. By comparing this RTN background risk to $\widetilde{y}$, one might be able to make a simple comparison how decisions will change. In other words, it might be easier to compare a decision made in the presence of an actual background risk against an RTN background risk than against no background risk. And by construction of the RTN class, the latter two yield the same optimal decisions. The rest of the paper provides a few examples of how RTN background risks can be used in different settings. 


\section{Mossin's Partial Myopia}

For the sake of clarity, we consider a particular choice problem; namely the choice of allocating wealth between a risky and a risk-free asset. To this end, let $w$ denote the initial wealth of the individual who must decide on the amount of wealth $a$ to invest in the risky asset, with the remaining wealth $w-a$ invested in the risk-free asset. The gross return on the risk-free asset is denoted as $R_{f} \geq 1$, whereas the risky asset's gross return is denoted by the random variable $\widetilde{R}$, where we assume that $\widetilde{R} \geq 0$ and $E \widetilde{R}>R_{f}$. This last assumption guarantees that the optimal investment in the risky asset $a *$ will be strictly positive.

The objective of the investor for a single period is to choose $a$ to maximize expected utility :

$$
\max E u(\widetilde{x}(a)) \equiv E u\left(w R_{f}+a\left(\widetilde{R}-R_{f}\right)\right)=\int_{0}^{\infty} u\left(w R_{f}+a\left(R-R_{f}\right)\right) d F(R),
$$

where $F$ denotes the distribution function for risky returns.

Mossin (1968a) considers a simple two-period dynamic portfolio problem under HARA preferences. The investor decides at date $t=0$ how to invest her wealth in a portfolio consisting of a risky asset and a risk-free asset. At the end of the first period, at date $t=1$, the investor can then optimally reinvest her realized wealth, i.e. she can "rebalance" her portfolio. At the end of the second period, at date $t=2$, the investor then realizes and consumes her final wealth. We assume that returns on the risky asset are statistically independent in each period and that the risk-free rate at date $t=1$, i.e. $R_{f}$, is known by the investor at date $t=0$.

The standard approach to solving such a problem over two periods requires a method such as dynamic programming and solving the program "backwards" in time. However, Mossin shows that the first-period investment decision can actually be solved assuming that one hundred percent of wealth will be invested in the risk-free asset at date $t=1$, which Mossin calls "partial myopia." Of course, in the special case where $R_{f}=1$ at date $t=1$, such as assumed in Gollier (2001), we then have complete myopia: the investment in the first period is the same as if no second period investment was available. 
Note that the ability to invest in both a risk-free and a risky asset at date $t=$ 1 is not without consequence. Investor welfare will increase when the risky asset is also available. But Mossin's Theorem tells us that the availability of the risky asset will not alter the investor's portfolio decisions made in the first period.

To establish Mossin's result using RTN background risk, we require the following Lemma, which is a well-known result and is proven, for example, in Gollier(2001, p. 58).

Lemma: Consider the solution to the standard portfolio problem (7) when preferences exhibit HARA. Let $\widehat{a}$ denote the solution to

$$
E\left[\left(1+B \widehat{a}\left(\widetilde{R}-R_{f}\right)\right)^{-\frac{1}{B}}\left(\widetilde{R}-R_{f}\right)\right]=0
$$

Then the solution to ( 7$)$ satisfies $a *=\widehat{a}\left(A+B w R_{f}\right)$.

We can now prove Mossin's result.

Theorem (Mossin): Consider the two-period investment problem under $H A R A$ utility, where the returns on the risky asset are independent between the two periods. At date $t=0$ the investor chooses an investment in the risky asset that is identical to the one she would choose if all wealth at date $t=1$ was invested in the risk-free asset.

Proof: Suppose the investor chooses her investment in the risky asset, $a_{0}$, at date $t=0$ under the assumption that all wealth will be re-invested at the risk-free rate. Let $\widetilde{w}_{1}$ be a random variable denoting her wealth at date $t=1$ under this investment strategy. Now, consider a change in her re-investment strategy to account for the opportunity to invest in a risky asset at date $t=1$. We let $\widetilde{R}_{2}$ denote the risky return for this risky asset and assume it is independent from the distribution of first-period returns, i.e. we assume that $\widetilde{w}_{1}$ and $\widetilde{R}_{2}$ are statistically independent random variables.

From the Lemma, it follows that the optimal re-investment in the risky asset at date $t=1$ is $a_{1}=\widehat{a}\left(A+B w_{1} R_{f}\right)$ for any realized wealth $w_{1}$. Viewed at date $t=0$, the investor's random wealth at date $t=2$ is thus

$$
\begin{gathered}
\widetilde{w}_{2}=\widetilde{w}_{1} R_{f}+a_{1}\left(\widetilde{R}_{2}-R_{f}\right) \\
=\widetilde{w}_{1} R_{f}+\widehat{a}\left(A+B \widetilde{w}_{1} R_{f}\right)\left(\widetilde{R}_{2}-R_{f}\right) .
\end{gathered}
$$


Compared to investing all proceeds in the risk-free asset, the additional risk for this re-investment strategy is thus $\left[\widehat{a}\left(\widetilde{R}_{2}-R_{f}\right)\right]\left(A+B \widetilde{w}_{1} R_{f}\right)$. Since $\widetilde{w}_{1}$ and $\widetilde{R}_{2}$ are independent, this additional risk is easily seen to be a RTN background risk of the form $\widetilde{z}(x)=(k+\widetilde{\varepsilon})\left(\frac{A}{B}+x\right)$, with $x \equiv w_{1} R_{f}, \widetilde{\varepsilon} \equiv \widehat{a} B\left(\widetilde{R}_{2}-E \widetilde{R}_{2}\right)$ and $k \equiv \widehat{a} B\left(E \widetilde{R}_{2}-R_{f}\right)$. Hence, at date $t=0$, maintaining the strategy of investing $a_{0}$ in the risky asset is still optimal. Q.E.D. ${ }^{5}$

It is interesting to note that we have no apparent background risks in Mossin's setting. Rather, we viewed $\widetilde{w}_{1} R_{f}$ as being optimal when future investment was all risk-free; and then we simply observed that allowing for future risky investment looked no different than adding a RTN background risk.

Our methodology can also be used to extend Mossin's Theorem to account for a risk-free rate at date $t=1$ that is stochastic, when viewed from date $t=0$. At date $t=0$, when the investor makes her initial portfolio decision, she does not know what the risk-free rate will be when she rebalances her portfolio at date $t=1$. A date $t=0$, the risk-free rate in the second period is viewed as random $\widetilde{R}_{f}$. To make our extension, we do require that $\widetilde{R}_{2}-\widetilde{R}_{f}$ is not only independent of $\widetilde{R}_{1}$, but that the excess return $\widetilde{R}_{2}-\widehat{R}_{f}$ is identical for each realized risk-free rate $\widehat{R}_{f} \cdot{ }^{6}$

To show that Mossin's result also holds in this case, we first note that $\widehat{a}$ in this case remains a constant. This follows by noting that $\widehat{a}$ in this case is defined as in (8), but using $\widetilde{R}_{2}-\widetilde{R}_{f}$. Thus $\widehat{a}$ is well defined and is independent of the realized risk-free rate $\widehat{R}_{f}$. At date $t=1$, the investor rebalances her portfolio as in equation (9) based on her realized risk-free rate, i.e.

$$
\widetilde{w}_{2}=\widetilde{w}_{1} \widehat{R}_{f}+\widehat{a}\left(A+B \widetilde{w}_{1} \widehat{R}_{f}\right)\left(\widetilde{R}_{2}-\widehat{R}_{f}\right) .
$$

The proof then proceeds as above, but with $k \equiv \widehat{a} B\left(E \widetilde{R}_{2}-\widehat{R}_{f}\right)$.

\footnotetext{
${ }^{5}$ Note that for the special case of constant relative risk aversion (CRRA), which requires $A=0$, we obtain complete myopia. This follows easily from the Lemma since $a_{1}$ is then simply a multiple of $w_{1}$, so that $a_{1} / w_{1}$ is constant, as is well known from Merton (1971).

${ }^{6}$ So, for example, if the risk-free rate at date $t=1$ is 100 basis points higher, the distribution for $\widetilde{R}_{2}$ concurrently shifts up by 100 basis points as well.
} 


\section{Portfolio Choice and Taxes}

In many circumstances, portfolio wealth is modified by some exogenous effect. One example is taxes. In this section, we show how our RTN background risks can be used to re-examine and extend an age-old problem in public finance: the question of how taxes affect investment in risky assets. Although there has been much research on the effects of taxes on portfolio choice over the years, most of it has focussed on the effects of differing tax rates for different asset classes. But some early research has focused on the effects of a simple flat-rate income tax on portfolio choice. Here we consider a theoretical model where there is a fixed tax rate on one's income. We show how, under fairly broad circumstances, an income tax will increase the investment in the risky asset. Unlike Mossin (1968b) and others, who assume that the income tax is only on earnings above the risk-free rate, we assume that the tax also applies to one's risk-free earnings. We extend the extant results further by considering a model with a random tax rate: the case where the tax rate is uncertain at the time portfolio choices are made.

Domar and Musgrave (1944) were the first to consider this problem. They argued, against conventional wisdom at the time, that income taxes were most likely to increase investment in risky assets, rather than decrease it. The basic model was formalized in an expected-utility setting by Mossin (1968b) and by Stiglitz (1969). ${ }^{7}$

Consider first the case of a wealth tax, with tax rate $t$. That is, the individual must pay a fixed percent of her final wealth as a tax. We analyze this wealth tax first, and then use our results to consider an income tax.

To analyze the effect of a wealth tax by using a RTN background risk, we proceed using three basic steps:

(1) starting from a world with no taxes, we first make an "appropriate choice" of RTN background risk $z(x),{ }^{8}$

\footnotetext{
${ }^{7}$ See Sandmo (2010) for an excellent summary, discussion and extension of these early results.

${ }^{8}$ Although $z(x)$ is deterministic for a given wealth $x$, we retain the terminology "background risk," since $z(\widetilde{x})$ is stochastic, once $\widetilde{x}$ is random.
} 
(2) we apply the fact that portfolio choices made with wealth $\widetilde{x}+\widetilde{z}$ are no different than those made with wealth $\widetilde{x}$, and

(3) we then compare choices made in a world with taxes to $\widetilde{x}+\widetilde{z}$.

This third step is hopefully easier than comparing choices in a world with taxes to $\widetilde{x}$ alone.

To implement step 1 in our analysis, consider the following RTN background "risk": $z(x)=-t\left(\frac{A}{B}+x\right)$, where $0<t<1$. In other words, we let $\widetilde{\varepsilon}$ be identically zero and define $k \equiv-t$. Although $t$ is simply a parameter in this definition, we eventually will want $t$ to denote the tax rate, when we move to step 3. Indeed, we choose this particular $z(x)$ because it differs in absolute value from the wealth tax $t x$ by a constant.

Since $0<t<1$, our constraint that $(1+k+\varepsilon)>0$ is trivially satisfied. Obviously, in this example $z$ is not random for a fixed level of wealth $x$. But since $z(\widetilde{x})$ varies with $\widetilde{x}$, we will still refer to $z$ as a RTN "background risk," since $z$ satisfies our definition (3).

In this case we obtain

$$
E u(\widetilde{x}+\widetilde{z})=(1-t)^{1-\frac{1}{B}} \cdot E u(\widetilde{x}) .
$$

Note that we can write total random wealth in this case as

$$
\widetilde{x}+\widetilde{z}=\widetilde{x}(1-t)-t \frac{A}{B} .
$$

Recall that, by construction, as is clear from (11), the optimal choice of investment in the risky asset $a *$ is the same both with and without the background risk $\widetilde{z}$. This is step 2 .

We can now make the step 3 comparison, which is quite simple. Using (12) we can write after-tax wealth as follows:

$$
\widetilde{x}(1-t)=(\widetilde{x}+\widetilde{z})+t \frac{A}{B} .
$$

We assume for now that $B>0$ and first consider the case where $A=0$, so that we have CRRA. In this case, we see that $\widetilde{x}(1-t)=(\widetilde{x}+\widetilde{z})$, so that 
there is no effect of a wealth tax on the optimal portfolio choice. That is, the optimal investment in the risky asset $a *$ is unchanged by the wealth tax. This effect is as expected under CRRA since wealth is reduced proportionally at every final wealth level.

Now suppose that $A>0$. Starting from wealth $\widetilde{x}+\widetilde{z}$, it follows from (13) that we add the positive constant $t \frac{A}{B}$ to every wealth level. But this will increase the optimal risky investment $a *$, since $B>0$ implies that absolute risk aversion is decreasing (DARA). Hence, the total effect of the wealth tax is to increase investment in the risky asset. This three-step procedure does not require us to directly compare a situation with the tax to one with no tax; rather we compare after-tax wealth to the RTN alternative $\widetilde{x}+\widetilde{z}$.

At first thought, it might seem like this result of increasing investment in the risky asset is simply due to DARA. However, DARA is neither necessary nor sufficient for this result to hold. The result does not hold in general for any DARA utility, unless utility belongs to the HARA class. Moreover consider the case where we allow $B<0$, such as the case with quadratic utility $(B=-1)$. Since $A+B x>0$, we obviously must have $A>0$. In this case, we have increasing absolute risk aversion. Now the term $t \frac{A}{B}$ in (13) above is negative; but due to the increasing risk aversion, it follows from the above reasoning that we once again have an increase in the investment in the risky asset, $a *$.

But what happens if $A<0 ?^{9} \quad$ Since we must have $B>0$ in this case, we once again have DARA. However, in this case $t \frac{A}{B}<0$. Thus, it follows from (13) and step (3) above that investment in the risky asset will actually decrease with the wealth tax. Thus we see that for DARA, $B>0$, the effect of a wealth tax depends critically on whether $A>0, A=0$, or $A<0$.

These results illustrate a Proposition by Stiglitz (1969, Proposition 1): A proportional wealth tax will increase [decrease, not affect] investment in the risky asset if relative risk aversion is increasing [decreasing, constant].

Suppose now that instead of a wealth tax, we have a flat rate income tax,

\footnotetext{
${ }^{9}$ Recall that, for HARA preferences $A<0$ corresponds to preferences exhibiting decreasing relative risk aversion, whereas $A>0$ corresponds to increasing relative risk aversion.
} 
with the same tax rate $t$. We do assume, as in Domar and Musgrave (1944) and in Mossin (1968b), that there is a full loss offset. ${ }^{10}$ In this case, since the tax is only on earned income and not on the initial wealth $w$ as well, it follows from (12) that we can write after-tax wealth as simply refunding the tax on initial wealth in equation (13)

$$
\widetilde{x}(1-t)+t w=(\widetilde{x}+\widetilde{z})+t\left(\frac{A}{B}+w\right) .
$$

We see from (14) that after-tax wealth differs from the case of a wealth tax only by the positive constant $t w$.

Suppose that we once again restrict $B>0$, so that we have DARA. If we also have $A>0$, it follows that $a *$ will be higher than it would be with no tax. Indeed, the investment in the risky asset is even higher than it would be in the case where $t$ was a tax on total wealth, not just on income. But reconsider now the case where $A<0$. Since $A+B w>0$ by assumption, the term $t\left(\frac{A}{B}+w\right)$ must be positive. Hence investment in the risky asset will increase. Even though a flat-rate wealth tax of $t$ would lessen the investor's investment in the risky asset, a flat-rate income tax of $t$ would increase such investment.

For the case where $B<0$, with increasing absolute risk aversion, whether $a *$ is lower or higher than with no tax once again depends upon the sign of $t\left(\frac{A}{B}+w\right)$. But since we restrict $A+B x>0$ for all $x$, the term $t\left(\frac{A}{B}+w\right)$ must be negative when $B<0$. Hence, a flat-rate income tax will cause $a *$ to rise in this case as well, compared to the case with no tax. However, increasing absolute risk aversion implies that the extra investment in the risky asset will be less with the income tax than it would be with a proportional wealth tax.

Note that, unlike Mossin (1968b), we did not assume that the risk-free rate was zero. Our income tax thus applies not only to a tax on returns above a risk-free return, but rather to a tax on the risk-free interest as well. Although with an unspecified utility representation, the tax on the risk-free interest can

\footnotetext{
${ }^{10}$ Stiglitz (1969) makes the need of this assumption very transparent. Suppose that the income tax rate $t$ is close to one with no loss offset. Then an investor would keep only a very small part of any investment gains, yet would be fully responsible for any investment losses. This would obviously deter investment in the risky asset.
} 
cause problems, as described by Sandmo (2010). In particular, although a substitution effect for the income tax always increases risky investment, a tax on the risk-free interest will cause a type-of income effect, that will reduce risky investment in the case of DARA $(B>0)$. Our specification of HARA utility resolves such issues in the current setting.

Under HARA utility, an income tax will always lead to an increase in risky investment. We can now extend the tax model to a setting in which the income tax rate is random. In other words, suppose that the investor must make her investment choice before she knows exactly how high the tax rate will be. She only knows the distribution of possible tax rates.

To this end, let $\widetilde{\varepsilon}$ be a zero-mean random variable that is statistically independent from portfolio returns. For any realized value of $\varepsilon, t+\varepsilon$ denotes the tax rate. Obviously here $t$ denotes the expected tax rate at the time the portfolio decision is made. We further assume that $0<t+\widetilde{\varepsilon}<1$ with probability one - the tax rate that is finally announced must be between zero and 1. The problem facing the investor is that she must allocate her wealth between the risky and the risk-free asset prior to observing the realization of $\widetilde{\varepsilon}$. In this case, we will show how it follows from the above analysis that more wealth is invested in the risky asset than would be invested with no taxes.

For any fixed tax rate $t+\varepsilon$ define $U(a, \varepsilon) \equiv E u(\widetilde{x}(a)(1-(t+\varepsilon))+t w)$ as the investor's expected utility, net of her flat-rate income tax, for a given $a$ and a given $\varepsilon$. The first-order condition for the optimal investment at this fixed tax rate is

$$
\frac{\partial U(a, \varepsilon)}{\partial a}=0
$$

which we assume to be satisfied at investment level $a_{\varepsilon}$. It is easy to show that $U(a, \varepsilon)$ is concave in $a$ for all values of $a$-not just at the optimal $a *$ From our analysis above, we know that for any $\varepsilon$ such that $0<t+\varepsilon<1$ we must have $a_{\varepsilon}>a_{N}$, where $a_{N}$ denotes the optimal investment in the risky asset when there are no taxes. This in turn implies that $\frac{\partial U(a, \varepsilon)}{\partial a}>0$ when evaluated at $a_{N}$ for every $\varepsilon$, due to the concavity of $U(a, \varepsilon)$.

For a random tax rate $t+\widetilde{\varepsilon}$, the first-order condition for portfolio choice 
becomes

$$
\int_{-t}^{1-t} \frac{\partial U(a, \varepsilon)}{\partial a} d G(\varepsilon)=0,
$$

where $G$ denotes the distribution function for $\widetilde{\varepsilon}$, which is known to the investor at the time of her portfolio decision. Let $a *$ denote the solution to (16). We cannot have $a * \leq a_{N}$, since this would imply that $\frac{\partial U(a, \varepsilon)}{\partial a}<0$ for every $\varepsilon$, so that (16) cannot hold. Hence, more is invested in the risky asset with the random income tax than would be invested with no income tax, $a *>a_{N}$.

Finally, we note that the methodology used above for a random income-tax rate would also apply for a random wealth tax, so long as we maintain our assumption that $0<t+\varepsilon<1$. Indeed, one can easily see how other similar scenarios are possible. For example, this methodology will also apply if we have random inflation that is independent of risky-asset returns. We just need to use a price deflator in place of a proportional wealth-tax rate (as long as we allow only for inflation, with no chance of deflation, i.e. as long as the price deflator remains larger than unity). This allows us to conclude under HARA that, compared to the case with no inflation, a random rate of inflation will cause the investor to increase [not change; decrease] investment in the risky asset if we have increasing [constant; decreasing] relative risk aversion. $^{11}$

\section{Portfolio Choice and Risk Vulnerability}

We now consider the risk vulnerability model in Gollier and Pratt (1996). They consider a background risk $\widetilde{y}$ with a non-positive mean that is independent of random wealth. They examine conditions under which this background risk induces less risk taking behavior. In the context of our portfolio problem, this would imply reducing investment in the risky asset. Although the conditions on preference that are equivalent to inducing this type of behavior are quite strong, Gollier and Pratt also present two sufficient conditions for this behavior, both of which are satisfied by HARA

\footnotetext{
${ }^{11}$ Of course, this is a very simplified model of inflation. See, for example, Brennan and Xia (2002) for a more complex model, similar in spirit to the result shown here.
} 
utility whenever $B>0 .{ }^{12}$ However, even these sufficient conditions are not particularly easy to interpret in terms of economic intuition. By choosing an appropriate RTN background risk, we show below that the Gollier-Pratt independent background risk is larger in the low-return states and smaller in the high-return states of nature. The intuition as to why investment in the risky asset decreases then becomes apparent.

To set up this argument, note that the first-order condition to (7) under HARA can be written as follows:

$$
\begin{gathered}
0=E\left[(A+B \widetilde{x}(a))^{-\frac{1}{B}}\left(\widetilde{R}-R_{f}\right)\right] \\
\equiv \int_{0}^{R_{f}}(A+B x)^{-\frac{1}{B}}\left(R-R_{f}\right) d F(R)+\int_{R_{f}}^{\infty}(A+B x)^{-\frac{1}{B}}\left(R-R_{f}\right) d F(R),
\end{gathered}
$$

where $x(a) \equiv w R_{f}+a\left(R-R_{f}\right)$. We assume that (17) is satisfied at some positive finite value $a *$. Note that the first integral on the left-hand side in (17) is negative, representing the marginal utility cost of a higher $a$ when returns are low, whereas the second term is positive, representing the marginal utility benefit of a higher $a$ when returns are high. The sufficient secondorder condition for a maximum is trivially satisfied, since expected utility is concave in $a$.

We simplify the Gollier and Pratt (1996) set up slightly by considering a zero mean background risk $\widetilde{y}$. The case with a negative mean can be derived in a quite straightforward manner by adding a negative constant to $\widetilde{y}$. To examine the risk-vulnerability result of Gollier and Pratt (1996), define $\widetilde{\varepsilon}$ independent of $\widetilde{R}$ with $E \widetilde{\varepsilon}=0$ implicitly via

$$
\widetilde{y}=\widetilde{\varepsilon}\left[\frac{A}{B}+w R_{f}\right] .
$$

Now we can apply the same three-step process used to analyze investment under taxes. We first define the RTN background risk $\widetilde{z}=\widetilde{\varepsilon}\left[\frac{A}{B}+x\right]$. We

\footnotetext{
${ }^{12}$ One sufficient condition for risk vulnerability is that preferences satisfy "standard risk aversion," as defined by Kimball (1993), which is characterized by decreasing absolute risk aversion and decreasing absolute prudence. That is, both absolute risk aversion $\frac{-u^{\prime \prime}(x)}{u^{\prime}(x)}$ and absolute prudence $\frac{-u^{\prime \prime \prime}(x)}{u^{\prime \prime}(x)}$ are decreasing in $x$. Another sufficient condition is that absolute risk aversion is both decreasing and convex in wealth.
} 
see that $\widetilde{z}$ simply replaces $w R_{f}$ with $x$ when compared to $\widetilde{y}$. Thinking of $\widetilde{\varepsilon}$ as white noise, we see that $\widetilde{y}$ has more noise than $\widetilde{z}$ when returns are low $\left(R<R_{f}\right.$, equivalently $\left.x<w R_{f}\right)$ and that $\widetilde{y}$ has less noise than $\widetilde{z}$ when returns are high $\left(R>R_{f}\right.$, equivalently $\left.x>w R_{f}\right)$.

If we add any RTN background risk of the form (3) with $k=0$, the first-order condition becomes

$$
\begin{aligned}
& 0=E(1+\widetilde{\varepsilon})^{1-\frac{1}{B}} E\left[(A+B \widetilde{x})^{-\frac{1}{B}}\left(\widetilde{R}-R_{f}\right)\right] \\
& =E\left[(1+\widetilde{\varepsilon})(1+\widetilde{\varepsilon})^{-\frac{1}{B}}\right] E\left[(A+B \widetilde{x})^{-\frac{1}{B}}\left(\widetilde{R}-R_{f}\right)\right] \\
& =\left[1+\frac{\operatorname{cov}\left(\widetilde{\varepsilon},(1+\widetilde{\varepsilon})^{-\frac{1}{B}}\right)}{E(1+\widetilde{\varepsilon})^{-\frac{1}{B}}}\right] E(1+\widetilde{\varepsilon})^{-\frac{1}{B}} E\left[(A+B \widetilde{x})^{-\frac{1}{B}}\left(\widetilde{R}-R_{f}\right)\right] \\
& =\left[1+\frac{\operatorname{cov}\left(\widetilde{\varepsilon},(1+\widetilde{\varepsilon})^{-\frac{1}{B}}\right)}{E(1+\widetilde{\varepsilon})^{-\frac{1}{B}}}\right] E\left[(A+B(\widetilde{x}+\widetilde{z}))^{-\frac{1}{B}}\left(\widetilde{R}-R_{f}\right)\right] .
\end{aligned}
$$

Our assumption that $1+k+\varepsilon>0$ implies that the constant first term on the left-hand side of the last line in (19) must be positive, so that the second term must be zero. Hence, we can re-write the first-order condition as

$$
\begin{gathered}
\int_{0}^{R_{f}} \int_{-\infty}^{+\infty}\left((A+B(x+z))^{-\frac{1}{B}}\left(R-R_{f}\right) d G(\varepsilon) d F(R)\right. \\
+\int_{R_{f}}^{\infty} \int_{-\infty}^{+\infty}\left((A+B(x+z))^{-\frac{1}{B}}\left(R-R_{f}\right) d G(\varepsilon) d F(R)=0,\right.
\end{gathered}
$$

where $G$ once again denotes the distribution function for $\widetilde{\varepsilon}$. From our previous argument about RTN background risk, this yields the same optimal investment in the risky asset, $a *$ as would hold with no background risk from (17). Thus we only need to complete the third step in our process, namely compare $\widetilde{x}+\widetilde{y}$ with $\widetilde{x}+\widetilde{z}$.

Since we assume $B>0$, it follows that

$$
\int_{-\infty}^{+\infty}\left((A+B(x+y))^{-\frac{1}{B}} d G(\varepsilon)>[<] \int_{-\infty}^{+\infty}\left((A+B(x+z))^{-\frac{1}{B}} d G(\varepsilon)\right.\right.
$$

for each $R<[>] R_{f}$. The fact that $u^{\prime}$ is convex allows us to use Jensen's inequality to compare $\widetilde{y}$ and $\widetilde{z}$, i.e. to compare the terms containing $\widetilde{\varepsilon}$. To see this more clearly, note that the multiplicative factor on the $\widetilde{\varepsilon}$ term is either $w R_{f}$ or wealth $x$; and since $a *$ is positive, the latter term is larger if and only if $R>R_{f}$.

Calculating $\frac{d E u}{d a}$ as in (20) but with $y$ replacing $z$, it follows that the negative term (marginal costs) is more negative and the positive term (marginal 
benefit) is less positive, when evaluated at $a *$. Hence, the optimal level of investment in the risky asset will fall, as expected. In other words, compared to its risk-taking-neutral counterpart, which changes marginal utility by a proportional amount everywhere, the independent background risk $\widetilde{y}$ increases marginal utility (and hence increases marginal costs) when returns are low and it decreases marginal utility (and hence decreases marginal benefits) when returns are high.

This result is easy to interpret when we consider what is going on in step 3. Compared to the RTN background risk $\widetilde{z}$, the actual background risk $\widetilde{y}$ is larger in the low-wealth states and smaller in the high-wealth states. This will increase the marginal utility cost and simultaneously decrease the marginal utility benefit of a higher level of risky investment, leading to a reduction of $a *$.

Although we examined the simplified case of $E \widetilde{y}=0$, it is straightforward to extend the analysis in the section to the case where $E \widetilde{y}<0$. To this end, simply replace (18) implicitly defining both $k<0$ and $\widetilde{\varepsilon}$ via $\widetilde{y}=(k+$ $\widetilde{\varepsilon})\left[\frac{A}{B}+w R_{f}\right]$. We can then proceed as above to verify that the Gollier and Pratt (1996) result also holds in this case. Essentially, a negative $k$ reduces wealth everywhere, which under DARA $(B>0)$ implies that the individual is more risk averse and thus reduces $a *$ further still.

In the next section, we provide a somewhat broader context in which we can apply the type of benchmarking result used here. In particular, we consider a linear mix of both an additive and a multiplicative background risk, and we show how such background risk can be easily compared to a particular RTN background risk.

\section{Linearly Dependent Background Risks}

Although our RTN class of background risks might be useful directly, as was the case with Mossin's Theorem, it is more likely to be useful as a benchmark, as was the case for decision making with income taxes or decision making with an independent background risk. In these cases, it may be easier to compare optimal decisions made in the presence of some external influences 
to the RTN case of $\widetilde{x}+\widetilde{z}$, rather than to $\widetilde{x}$ directly. Below we provide a more general proposition and we show how several of our previous results follow from it.

We assume once again that the investor has decreasing absolute risk aversion $(B>0)$. The investor now makes a portfolio decision in the face of a linearly dependent background risk $\widetilde{y}$, where $\widetilde{y}$ takes the form:

$$
\tilde{y}(x)=(\alpha+\beta x)(k+\tilde{\varepsilon}),
$$

with the zero-mean random variable $\tilde{\varepsilon}$ being distributed independently of $x$ and with $k \leq 0$. We assume that $\beta \geq 0, k \leq 0$ and $(\alpha+\beta x) \geq 0$ over the relevant range of wealth. Note that our parameter assumptions imply that, as wealth $x$ increases, the mean of the background risk $\tilde{y}(x)$ either remains at zero or becomes more negative. At the same time, as wealth $x$ increases, the variability of $\tilde{y}(x)$ either remains unchanged or increases. Thus, the background risk "worsens" as the investor's wealth $x$ increases. Note that when $\alpha=0$, the background risk is a purely multiplicative background risk.

We are now set to prove the following result.

Proposition 1 Consider a background risk of the form (22). Utility is $H A R A$ of the form (1) with $B>0$ (DARA).

(i) If $\alpha / \beta=A / B$, then optimal investment in the risky asset is unchanged by $\tilde{y}(x)$.

(ii) If $\beta=0$, then $\tilde{y}(x)$ decreases the optimal investment in the risky asset. (iii) For $\beta>0$ and $\alpha / \beta \neq A / B$, if $W /\left[\left(A-\frac{\alpha}{\beta} B\right)+B x W\right]>1 \forall W$, then $\tilde{y}(x)$ decreases [increases] the optimal investment in the risky asset if and only if

$$
\frac{\alpha}{\beta}>[<] \frac{A}{B} \text {. }
$$

$\underline{\text { Proof }}$

If $\alpha / \beta=A / B$, it is easy to see that $\widetilde{y}$ itself is risk taking neutral, which proves $(i)$.

Next consider the case where $\beta=0$. This is the risk vulnerability model of Gollier and Pratt(1996), which we have already examined in the previous section. Thus, (ii) holds. 
Thus we need to only examine the case where $\alpha / \beta \neq A / B$ for $\beta>0$. As "step 1," our canonical choice of $\widetilde{z}(x)$ is simply to replace $(\alpha, \beta)$ with $\left(\frac{A}{B}, 1\right)$ in (22). We can easily rewrite $\widetilde{y}(x)$ as a mixture of an additive and a multiplicative background risk. From (22), it follows that

$$
\widetilde{W} \equiv x+\widetilde{y}(x)=-\frac{\alpha}{\beta}+\left(\frac{\alpha}{\beta}+x\right)(1+\beta(k+\tilde{\varepsilon})) .
$$

Hence,

$$
A+B \widetilde{W}=A+B\left[-\frac{\alpha}{\beta}+\left(\frac{\alpha}{\beta}+x\right)(1+\beta(k+\tilde{\varepsilon}))\right]=A^{0}+B x^{0} \widetilde{\kappa}
$$

where

$$
\begin{aligned}
A^{0} & \equiv A-\frac{\alpha}{\beta} B \\
x^{0} & \equiv \frac{\alpha}{\beta}+x \\
\widetilde{\kappa} & \equiv 1+\beta(k+\widetilde{\varepsilon}) .
\end{aligned}
$$

Since we have HARA utility, we can replace $u^{\prime}(W)=(A+B W)^{-1 / B}$ with $u^{\prime}\left(w^{0}\right)=\left(A^{0}+B w^{0}\right)^{-1 / B}$, where $w^{0} \equiv x^{0} \kappa$, and apply known results about multiplicative background risks. In particular, our hypothesis assumes that

$$
R R A_{0}(x)=\frac{W}{A^{0}+B W}>1,
$$

for the support of $W$. Hence, the relative risk aversion of the latter utility function, with $A$ replaced by $A^{0}$ is greater than 1 ,

$$
R R A_{0}\left(w^{0}\right) \equiv \frac{w^{0}}{A^{0}+B w^{0}}>1,
$$

for the support of $w^{0}$. The case with a mixture of an additive and multiplicative background risk $x+\widetilde{y}$ can thus be transformed into a model with a purely multiplicative background risk $x^{0} \widetilde{\kappa}$, via our transformed HARA, utility,with $A$ replaced by $A^{0}$.

Note that the investor's optimization problem (7) can be rewritten as

$$
\max E\left[\frac{1}{1-B}(A+B(\widetilde{x}(a)))^{1-1 / B}\right]=E\left[\frac{1}{1-B}\left(A^{0}+B\left(\widetilde{x}^{0}(a)\right)\right)^{1-1 / B}\right] .
$$


From $(27)$, it follows that adding $\widetilde{y}(x)$ to the optimal portfolio wealth $\widetilde{x}(a)$ is equivalent to multiplying $\widetilde{x}^{0}(a)$ by the purely multiplicative background risk $\widetilde{\kappa}$ and using the modified HARA utility function, with $A$ replaced by $A^{0}$. If $A^{0}<0$, equivalently if $\frac{\alpha}{\beta}>\frac{A}{B}$, then $R R A_{0}(W)$ is decreasing in $W$. It then follows from Corollary 3 in Franke et al. (2006) that the optimal investment in the risky asset will decrease. On the other hand, if $A^{0}>0$, i.e. if $\frac{\alpha}{\beta}<\frac{A}{B}$, then $R R A_{0}(W)$ is increasing in $W$. In this case Corollary 3 in Franke et al. (2006) implies that optimal investment in the risky asset increases. This proves $($ iii).

Note that the condition for part (iii) in the Proposition, $W /\left[A^{0}+B W\right]>1$ $\forall W$ limits the range of $W$ in part (iii) based on a transformed HARA utility function, where we replace $A$ with $A^{0}$. We also note that the Gollier and Pratt (1996) result is part (iii) of our Proposition. Also, if $\alpha=0$ we have the case where $\widetilde{y}(x)$ is a pure multiplicative background risk, as examined in more detail by Franke et al. (2006).

Although our proof of Proposition 1 is for the strict HARA class, the result also holds for the case of CARA utility $(B \rightarrow 0)$. In particular, if we also allow $\beta \rightarrow 0$, we see that $(i)$ holds in the limit, and $a *$ is unchanged, as is well known for CARA preferences with an independent additive background risk. If $\beta>0$, the we must have $\alpha / \beta<A / B$ as $B \rightarrow 0$, so that $a *$ increases. With $\beta>0$, the background risk is larger for states with higher market returns. This leads to a shifting of even more wealth to high return states via an increase in $a *$.

Note that all of our tax results from section 4 follow from part (iii) of Proposition 1, so long as $B>0$ and so long as wealth is restricted to a range where $R R A_{0}>1$, as required in the Proposition. To see this, we set $\widetilde{\varepsilon}$ identical to zero and set $k=-t$. If there is wealth tax, we can define $\alpha=0$ and $\beta=1$. Thus $x+y=x(1-t)$. Moreover, $0=\frac{\alpha}{\beta}<[>,=] \frac{A}{B}$ if $A>[<,=] 0$. Hence, we obtain the Proposition from Stiglitz (1969) as described in section 4.

If there is an income tax instead of a wealth tax, we proceed as above, but set $\alpha=-w$. Thus $x+y=x(1-t)+t w$. In this case $\frac{\alpha}{\beta}=-w$, which is always smaller than $\frac{A}{B}$ since we assume $A+B w>0$. Hence, risky investment always increases. Both of these tax results also follow for a random tax rate, by simply allowing $\widetilde{\varepsilon}$ to be non-degenerate. 


\section{A Numerical Illustration}

Here we provide a simple example to illustrate Proposition 1 . Consider a market with three equally likely states for $R_{m}$. In the bottom state, $R_{m}-$ $R_{f}=-0.15$. In the middle state, $R_{m}-R_{f}=0$ and in the top state, $R_{m}-R_{f}=0.3$. The background risk is linear in the market return and given by $y(x)=(\alpha+\beta x)[(-0.1)+\varepsilon]$ with $\varepsilon=+0.3-$ or $\varepsilon=0.3$, each with an equal probability.

The Proposition says that risk taking increases (decreases) according to whether the ratio of the background risk parameters, $\alpha / \beta$ is less than (greater than) the ratio of the HARA parameters $A / B$. We set $A=-5$ and $B=0.5$, which implies that the critical benchmark ratio is $A / B=-10$. The individual has an initial wealth of $w=100$.

In the first column of Table 1, we show the base case with no background risk, with $\alpha=\beta=0$. In this case the optimal investment is to invest $a *=\$ 75.25$ into the risky stock, with the rest of initial wealth invested in the risk-free bond. Obviously, in the state where $R_{m}-R_{f}=0$, the amount invested in the risky stock has no effect on final wealth. If $R_{m}-R_{f}<0$ $[>0]$, final wealth will be lower [higher] and marginal utility of wealth will be higher [lower] than when $R_{m}-R_{f}=0$. In particular, from column 1 , we see that marginal utility of wealth is $29.6 \%$ higher when $R_{m}-R_{f}<0$ (the marginal cost state for increasing $a *$ ) than when $R_{m}-R_{f}=0$. That is, $v^{\prime}(W) / v^{\prime}\left(w R_{f}\right)=1.296$. We also see that the marginal utility of wealth is only $64.8 \%$ as high when $R_{m}-R_{f}>0$ (the marginal benefit state for increasing $a *$ ) than when $R_{m}-R_{f}=0$. At $a *=\$ 75.25$, the marginal benefits equal the marginal costs for changes in stock investment.

In all of our cases, except for the base case, we will have marginal utility in the presence of background risk $\widetilde{y}(x)$ higher than marginal utility with no background risk. This will occur, for example in case 2 , with $\alpha=-10$ and $\beta=1$. In this case, the background risk $\widetilde{y}(x)$ is itself a RTN background risk. Even though the marginal utility of wealth will be higher at every wealth level for the derived utility function $v$, it will change proportionately at all wealth levels. In the second column of Table $1, v^{\prime}(W) / v^{\prime}\left(w R_{f}\right)$ simply compares how marginal utility changes in the up-state and in the down state, relative to $R_{m}-R_{f}=0$. As expected, the relative changes for a RTN background 
risk are no different than for the case with no background risk.

In cases $3-5$, our Proposition says that $a *$ should fall compared to the base case, since $\alpha / \beta>-10$. Case $3(\alpha=0, \beta+0.5)$ illustrates a purely multiplicative background risk, while cases 4 and 5 are a particular mix of an additive and a multiplicative background risk. ${ }^{13}$ In all three case, for the state where $R_{m}-R_{f}<0$ we see that the marginal utility increases more than the $29.6 \%$ for the base case, implying a higher weight on the (negative) costs. Likewise, for the state where $R_{m}-R_{f}>0$ we see that the marginal utility is reduced more than the reduction to $64.8 \%$ for the base case. This implies a lower weight on the marginal benefits. It thus follows in all three cases that $a *=\$ 75.25$ is too high, and expected utility can be increased by reducing $a *$.

Case 6 in Table 1 is an example where $\alpha / \beta<-10$ and hence our analysis of relative changes to weighting of marginal benefits and marginal costs is reversed, when compared to cases 3-5. Hence $a *=\% 75.25$ is too low, the optimal investment in stock should increase, compared to the base case.

Table 1: Linearly Dependent Background Risk and Stock Investment

\begin{tabular}{|c|c|c|c|c|c|c|}
\hline Parameters & $(1)$ & $(2)$ & $(3)$ & $(4)$ & $(5)$ & $(6)$ \\
$A=-5$ & $\alpha=0$ & $\alpha=-10$ & $\alpha=0$ & $\alpha=15$ & $\alpha=-5$ & $\alpha=-25$ \\
$B=0.5$ & $\beta=0$ & $\beta=1$ & $\beta=0.5$ & $\beta=0.5$ & $\beta=1$ & $\beta=0.5$ \\
\hline$\left.\frac{v^{\prime}}{v^{\prime}\left(w R_{f}\right)}\right|_{a=75.25}$ & & & & & & \\
$R_{m}-R_{f}<0$ & 1.296 & 1.296 & 1.314 & 1.320 & 1.318 & 1.273 \\
$R_{m}-R_{f}=0$ & 1.000 & 1.000 & 1.000 & 1.000 & 1.000 & 1.000 \\
$R_{m}-R_{f}>0$ & 0.648 & 0.648 & 0.635 & 0.631 & 0.632 & 0.669 \\
\hline$a^{*}$ & 75.25 & 75.25 & 72.17 & 63.36 & 74.09 & 80.54 \\
\hline
\end{tabular}

$\underline{\text { notes }}$

1. Background risk is $y(x)=(\alpha+\beta x)[(-0.1)+\varepsilon], \varepsilon= \pm 0.3$ with equal probability.

\footnotetext{
${ }^{13}$ See Franke et al. (2011).
} 
2. Utility is HARA with $u^{\prime}(w)=(A+B w)^{-1 / B}, A=-5, B=0.5$

3. $W \equiv x+y(x)$, with initial wealth $w=100$.

4. $a^{*}$ denotes the optimal investment in risky asset.

5. $R_{f}=1.03$ and $R_{m}-R_{f}=-15 \%, 30 \%, 0 \%$, with equal probability.

\section{Concluding Remarks}

We defined a parametric class of background risks for HARA utility that we call the class of risk-taking-neutral (RTN) background risks. These background risks affect overall satisfaction, but do not alter economic choices. In some cases, such as Mossin's (1968a) Theorem about dynamic investment strategy, we were able to show the result by directly linking wealth to a particular RTN background risk. In other cases, we were able to choose a particular RTN, and then easily compare an extant background risk to our chosen RTN counterpart.

The analysis generally proceeded by following three steps: (1) find an appropriate RTN background risk $\widetilde{z}(x),(2)$ note that decisions made in the presence of $\widetilde{z}(x)$ are identical to decisions made when no background risk is present, (3) compare decisions made in the presence of some actual background risk $\widetilde{y}(x)$ to those made in the presence of $\widetilde{z}(x)$. In some cases, such as Mossin's Theorem, step 3 was simply noting that $\widetilde{y}(x)$ was itself RTN. In other cases, such as when analyzing risk vulnerability, it was fairly easy to see that $\widetilde{y}(x)$ was riskier than [less risky than] $\widetilde{z}(x)$ in states of the world with low stock returns [high stock returns].

In addition to illustrating some known results, often with some additional intuition, we were able to extend some extant results. For example, we showed how Mossin's (1968a) partial myopia for dynamic investment strategies can also hold in world where future risk-free rates are not yet known. We also were able to allow for an income tax on all income, not just income above the risk-free return, and still get an increase in risky investment. Moreover, we extended these tax results to a model in which the tax rate is unknown (i.e. random) at the time investment decisions must be made. Finally, we 
presented a more general result about risk taking behavior in the presence of a linearly dependent background risk.

We wish to reiterate that our RTN class of background risks is not exhaustive in the sense that other background risks might also exhibit the property of not affecting decisions about the primary risk. Moreover, the RTN class of background risks as defined here is parametric, based upon the individual's HARA utility function. Still, we hope that the RTN class of background risks as defined in this paper can prove useful in deriving many new results, as well as better interpreting the intuition of many extant results. 


\section{References}

[1] Brennan, Michael J. and Xia, Yihong. "Dynamic Asset Allocation under Inflation," Journal of Finance 57, 2002, pp. 1201-1238.

[2] Campbell, John and Viceira, Luis. Strategic Asset Allocation, 2002, Oxford University Press: Oxford, UK.

[3] Cass, David and Stiglitz, Joseph. "The Structure of Investor Preferences and Asset Returns, and Separability in Portfolio Allocation," Journal of Economic Theory 2, 1970, pp. 122-160.

[4] Dana, Rose-Anne and Scarsini, Marco. "Optimal Risk Sharing with Background Risk," Journal of Economic Theory 133, 2007, pp. 152-176.

[5] Doherty, Neil and Schlesinger, Harris. "Optimal Insurance in Incomplete Markets," Journal of Political Economy 91, 1983, pp. 1045-1054.

[6] Domar, Evsey and Musgrave, Richard. "Proportional Income Taxation and Risk Taking," Quarterly Journal of Economics 58, 1944, pp. 388422.

[7] Franke, Guenter; Schlesinger, Harris and Stapleton, Richard. "Multiplicative Background Risk," Management Science 52, 2006, pp. 146-153.

[8] Franke, Guenter; Schlesinger, Harris and Stapleton, Richard. "Risk Taking with Additive and Multiplicative Background Risk," Journal of Economic Theory 146, 2002, pp. 1547-1568.

[9] Gollier, Christian. The Economics of Risk and Time. Cambridge: MIT Press, 2001.

[10] Gollier, Christian, and Pratt, John W. "Risk Vulnerability and the Tempering Effect of Background Risk." Econometrica, 64, 1996, pp. 1109-24.

[11] Kihlstrom, Richard; Romer, David and Williams, Steve. "Risk Aversion with Random Initial Wealth", Econometrica 49, 1981, pp. 911-920.

[12] Kimball, Miles S. "Standard Risk Aversion." Econometrica 61, 1993, pp. 589-611. 
[13] Merton, Robert. "Optimal Consumption and Portfolio Rules in a Continuous-Time Model," Journal of Economic Theory 3, 1971, pp. 373413.

[14] Mossin, Jan. "Optimal Multiperiod Portfolio Policies," Journal of Business 41, 1968a, pp. 215-229.

[15] Mossin, Jan. "Taxation and Risk-Taking: An Expected-Utility Approach," Economica 35, 1968b, pp. 74-82.

[16] Nachman, David. "Preservation of 'More Risk Averse' under Expectations." Journal of Economic Theory 28, 1982, pp. 361-368

[17] Ross, Stephen A. "Some Stronger Measures of Risk Aversion in the Small and in the Large with Applications," Econometrica 49, 1981, pp. 621-638.

[18] Sandmo, Agnar. "Uncertainty in the Theory of Public Finance," Geneva Risk and Insurance Review 35, 2010, pp. 1-18.

[19] Stiglitz, Joseph. "The Effects of Income, Wealth and Capital Gains Taxation on Risk Taking," Quarterly Journal of Economics 83, 1969, pp. 262-283. 